\title{
Antibody-nanoparticle conjugate constructed with trastuzumab and nanoparticle albumin-bound paclitaxel for targeted therapy of human epidermal growth factor receptor 2-positive gastric cancer
}

\author{
JIAN XIONG ${ }^{1,3^{*}}$, SHUHUA HAN $^{2 *}$, SHUANG DING $^{1}$, JINGCHAO HE $^{1}$ and HAIJUN ZHANG ${ }^{1}$ \\ Departments of ${ }^{1}$ Oncology and ${ }^{2}$ Respiration, Zhongda Hospital, Medical School of Southeast University, \\ Nanjing, Jiangsu 210009; ${ }^{3}$ Department of Hematology, Dongfeng General Hospital, \\ Hubei University of Medcine, Shiyan, Hubei 442000, P.R. China
}

Received June 17, 2017; Accepted December 21, 2017

DOI: 10.3892/or.2018.6201

\begin{abstract}
Gastric cancer (GC) is the most lethal malignancy in the digestive system. This study investigated an antibodynanoparticle conjugate (ANC) constructed with trastuzumab (Herceptin ${ }^{\circledR}$ ) and nanoparticle albumin-bound paclitaxel (nab-paclitaxel, Abraxane ${ }^{\circledR}$ ) (trastuzumab/nab-paclitaxel) as a novel strategy of targeted therapy for human epidermal growth factor receptor 2 (HER2) positive GC. The ANC was fabricated with trastuzumab and nab-paclitaxel by a 'one-step' synthesis using EDC/NHS. In vitro antitumor efficacy was evaluated by cell viability, apoptosis rate and cell cycle of HER2-positive GC NCI-N87 cells and compared with paclitaxel $\left(\right.$ Taxol $^{\circledR}$ ), nab-paclitaxel and trastuzumab/nab-paclitaxel. In addition, GC xenograft models were established to evaluate antitumor efficacy in vivo. These results demonstrated that trastuzumab/nab-paclitaxel was spherical with a suitable size $(139.18 \pm 32.06 \mathrm{~nm})$. The half-maximal inhibitory concentration $\left(\mathrm{IC}_{50}\right)$ for NCI-N87 cells was $0.24 \pm 0.08,0.13 \pm 0.03$ and $0.048 \pm 0.01 \mu \mathrm{g} / \mathrm{ml}$ of paclitaxel, nab-paclitaxel and trastuzumab/nab-paclitaxel, respectively. Compared with paclitaxel and nab-paclitaxel, trastuzumab/nab-paclitaxel could induce
\end{abstract}

Correspondence to: Professor Haijun Zhang, Department of Oncology, Zhongda Hospital, Medical School of Southeast University, 87 Dingjiaqiao Road, Nanjing, Jiangsu 210009, P.R. China

E-mail: haijunzhang@seu.edu.cn

*Contributed equally

Abbreviations: AGC, advanced gastric cancer; ANC, antibodynanoparticle conjugate; HER2, human epidermal growth factor receptor 2; T-DM1, ado-trastuzumab emtansine; HSA, human serum albumin; OS, overall survival; ADC, antibody-drug conjugates; GAPDH, glyceraldehyde-3-phosphate

Key words: antibody-nanoparticle conjugate, gastric cancer, human epidermal growth factor receptor 2 , targeted therapy a higher rate of apoptosis and significant G2/M arrest. At 4 weeks after treatment, tumor-bearing mice had a mean tumor volume of $233 \pm 24 \mathrm{~mm}^{3}$ treated by trastuzumab/nab-paclitaxel, $559 \pm 97 \mathrm{~mm}^{3}$ by nab-paclitaxel, $871 \pm 94 \mathrm{~mm}^{3}$ by paclitaxel and $1,576 \pm 190 \mathrm{~mm}^{3}$ by PBS as control, respectively, which showed that trastuzumab/nab-paclitaxel could surpass nab-paclitaxel and paclitaxel in antitumor effect. Furthermore, the NIR imaging indicated that trastuzumab/nab-paclitaxel labeled by NIR-797 could more precisely focus on tumor regions. In conclusion, trastuzumab/nab-paclitaxel could mediate targeted therapy and enhance antitumor efficacy, which could represent a novel therapeutic agent for HER2-positive GC.

\section{Introduction}

Gastric cancer (GC) is the most lethal malignancy in the digestive system $(1,2)$. Due to the asymptomatic nature, non-specific clinical manifestations, and the lack of efficient screening programs, most GC patients were diagnosised with advanced stages. The prognosis of patients with advanced GC (AGC) is still poor even after multidisciplinary therapy combined with surgery, chemotherapy, and radiation therapy (3). Human epidermal growth factor receptor 2 (HER2) is a member of a family of receptors acting as a proto-oncogene. HER2 is overexpressed in $\sim 20 \%$ of GC, and now it is an important target for GC. Trastuzumab, a monoclonal antibody that targets HER2, inhibits HER2-mediated signaling and thus induces antibody-dependent cellular cytotoxicity against tumor cells $(4,5)$. The introduction of trastuzumab made a new term in HER2-positive GC.

Trastuzumab combined with chemotherapy demonstrates a significant survival benefit in patients with HER2-positive AGC in the clinic. In ToGA study (trastuzumab in combination with chemotherapy versus chemotherapy alone for treatment of HER2-positive advanced gastric or gastro-esophageal junction cancer), an open-label, international, phase III, randomized controlled trial, patients with HER2-postive GC were randomly assigned to receive a standard chemotherapy regimen or chemotherapy in combination with trastuzumab. Results showed that those who received trastuzumab plus 
chemotherapy had prolonged overall survival (OS) (6). Furthermore, with the development of antibody-drug conjugate (ADC) technique, strategies which take advantage of selective delivery of anticancer drugs by monoclonal antibodies to antigen-expressing tumor cells have proved efficacy. For example, ado-trastuzumab emtansine (T-DM1) consists of trastuzumab linked to cytotoxic agent of emtansine as a single agent, which shows statistically significant antitumor efficacy and minor systemic toxicity in HER2-positive tumors (7-9). In GC, T-DM1 showed a promising antitumor efficacy in HER2-positive GC cell lines in vitro and xenograft tumors in vivo $(10,11)$. Nowadays, several clinical trials are underway in patients with HER2-positive AGC, including the efficacy and safety of T-DM1 compared with paclitaxel (PTX) (9). However, T-DM1 does not show survival benefit in HER2-positive AGC as the expected remarkable efficacy for HER2-positive breast cancer (12). Therefore, HER2-directed therapies for HER2-positive GC remains a challenge.

Fortunately, nanomedicine has a significant impact on cancer therapy with the introduction of drug delivery system based on nanobiotechnology, such as nanoparticle albumin-bound paclitaxel (nab-paclitaxel). Nab-paclitaxel is a solvent-free suspension of paclitaxel and human serum albumin (HSA) with an average mini size, which allows better delivery of paclitaxel into tumors via the unique mechanism (gp60-caveolin-1-SPARC) and passive targeting of EPR (enhanced permeability and retention) effect. To date, many studies have proved the superiority of nab-paclitaxel in tumors, including GC (13-18). However, its clinical success is stilled limited by unfavorable pharmacokinetics, suboptimal biodistribution and toxicities (19). Antibody-nanoparticle conjugate (ANC) offers opportunities for optimized targeted cancer treatment $(20,21)$. In view of these studies, here, the conjugation of the two clinical drugs of nab-paclitaxel and trastuzumab is presented as an ANC single-agent (trastuzumab/nab-paclitaxel) for precisly targeted therapy of HER2-positive GC.

\section{Materials and methods}

Main materials. Abraxane ${ }^{\circledR}$ [paclitaxel for injection (albumin bound), lot no. 6109342] was from Celgene (Fresenius Kabi USA, LLC Melrose Park, IL, USA). Taxol ${ }^{\circledR}$ (paclitaxel injection) was from Bristol-Myers Sguibb (Corden Phama Latina S.P.A, Sermoneta, Italy). Herceptin ${ }^{\circledR}$ (trastuzumab, lot no. ab2428) was purchased from Abcam. FBS (fetal bovine serum, lot no. 1698221) was purchased from Gibco Chemical Co. (Carlsbad, CA, USA). Pen Strep (penicillin streptomycin, lot no. 1665735), $0.25 \%$ trypsin-EDTA and PBS (phosphatebuffered saline, lot no. AAL211089) were purchased from HyClone (GE Healthcare Life Sciences). Matrigel basement membrane matrix (lot no. 356324) was from Corning Inc. (Corning, New York, NY, USA). EDC (C8H17N3HCL, $\mathrm{N}$-(3-dimethylaminopropyl)-N'-ethylcarbodiimide hydrochloride, cat. no. 25952-53-8) and NHS $\left(\mathrm{C}_{4} \mathrm{H}_{5} \mathrm{NO}_{2}\right.$, N-hydroxysuccinimide, cat. no. 6066-82-6) were from Sigma-Aldrich (St. Louis, MO, USA). Cell Counting Kit-8 (CCK-8) was purchased from Dojindo Laboratories, (Kumamoto, Japan). Annexin V-FITC/PI Apoptosis Detection kit was from Nanjing KeyGen Biotech Co. (Nanjing, China). Hematoxylin-eosin staining kit from Beyotime Institute of Biotechnology
(Shanghai, China). BALB/c nude mice (weighing 20-22 g, 5 weeks old, half were male and half female) were obtained from Comparative Medicine Centre, Yangzhou University (Yangzhou, Jiangsu, China). Monoclonal antibodies of Bax (cat. no. ssc-493), Bcl-2 (cat. no. sc-509), caspase-3 (cat. no. ssc-271759), caspase-8 (cat. no. sc-56071), caspase-9 (cat. no. sc-17784) and survivin (cat. no. sc-101433) was from Santa Cruz Biotechnology Inc. (Santa Cruz, CA, USA). NIR797-isothiocyanate (MW 880.14 Da) was purchased from Sigma-Aldrich Co. The transmission electron microscopic (TEM) images were obtained by a JEM-2100, JEOL Ltd. (Tokyo, Japan). Flow cytometry analysis was performed by BD FACSCalibur (BD Biosciences, San Jose, CA, USA). Cells were imaged using a confocal microscope (Zeiss LSM 710; Carl Zeiss, Oberkochen, Germany), and the tumor-bearing nude mice were imaged with a Caliper IVIS (in vivo imaging system; Perkin-Elmer, Waltham, MA, USA). All the experiments were conducted according to the manufacturer's protocols. The reagents were of analytical grade.

Preparation of ANC of trastuzumab/nab-paclitaxel. ANC of trastuzumab/nab-paclitaxel was synthesized using EDC/NHS by surface activation method. Briefly, $500 \mu \mathrm{l}$ of nab-paclitaxel was dissolved in $1 \mathrm{ml}$ of PBS followed by the addition of $100 \mu \mathrm{l}$ NHS $\left(5.75 \times 10^{-7} \mathrm{~g} / \mathrm{ml}\right)$ and $100 \mu 1 \mathrm{EDC}\left(2.3 \times 10^{-7} \mathrm{~g} / \mathrm{ml}\right)$. After spining at $10 \mathrm{rpm}$ for $120 \mathrm{~min}, 20 \mu 1$ of trastuzumab $(0.2 \mathrm{mg} / \mathrm{ml})$ was added in the suspension. After further rotation at $10 \mathrm{rpm}$ for $120 \mathrm{~min}$ at $4^{\circ} \mathrm{C}$, it was ultracentrifuged at $1 \times 10^{4} \mathrm{rpm}, 4^{\circ} \mathrm{C}$ for $15 \mathrm{~min}$ to remove excess EDC/NHS and unconjugated trastuzumab. The process was repeated 3 times after sonication. Further, the ANC of trastuzumab/nab-paclitaxel were resuspended in $1 \mathrm{ml} \mathrm{PBS}$ and stored at $-20^{\circ} \mathrm{C}$. Morphological characteristics of the ANC of trastuzumab/nab-paclitaxel were examined using a TEM. Dynamic light scattering (DLS) was performed to determine the hydrodynamic radius ( $\mathrm{Rh}$ ) of trastuzumab/nab-paclitaxel at $25^{\circ} \mathrm{C}$ using a Dynapro ${ }^{\mathrm{TM}}$ plate reader (Wyatt Technology, Santa Barbara, CA, USA).

Cell culture. The human HER2-postive GC cell line, NCI-N87, purchased from Cell Bank of Chinese Academy of Sciences (Shanghai, China) and were cultured in RPMI-1640 supplemented with $10 \%$ heat inactivated fetal bovine serum (FBS), $1 \%$ Pen Strep in a humidified incubator in $5 \% \mathrm{CO}_{2}$ at $37^{\circ} \mathrm{C}$.

Cell viability assay. NCI-N87 cells were seeded in 96-well plates at a density of $\sim 5,000$ cells per well and treated with different treatments, paclitaxel, nab-paclitaxel and trastuzumab/nab-paclitaxel, respectively. Cell without treatment were used as a control. After further incubation for $48 \mathrm{~h}$ at $37^{\circ} \mathrm{C}$, the relative cell viability was assessed using CCK- 8 assays. Briefly, after the different treatments, CCK-8 $(10 \mu \mathrm{l})$ was added to each well and incubated for a further $2 \mathrm{~h}$. Optical density (OD) at $450 \mathrm{~nm}$ was read on an ELx800 microplate reader (BioTek, Vermont, WI, USA), and then the cell viability was calculated as follows:

$$
\mathrm{OD}_{(450 \mathrm{~nm} \text { in test cells })} / \mathrm{OD}_{(450 \mathrm{~nm} \text { in control cells) }} \times 100 \%
$$

Cell cycle analysis. NCI-N87 cells were seeded in 6-well plates. After different treatments, cells were collected, fixed 
in $75 \%$ cold ethanol at $4^{\circ} \mathrm{C}$ for at least $2 \mathrm{~h}$, washed by cold phosphate-buffered saline, stained with PI/RNase staining buffer for $15 \mathrm{~min}$, and then measured by flow cytometry.

Apoptosis assay. Quantification of apoptotic cells was determined using an Annexin V-fluorescein isothiocyanate (FITC)/ propidum iodide (PI) detection kit (BD Pharmingen, San Diego, CA, USA) according to the manufacturer's instructions. Cells were collected after different treatments, washed twice with cold PBS and resuspended in $100 \mu \mathrm{l}$ binding buffer, followed by staining with $5 \mu \mathrm{l}$ of Annexin V-FITC and $10 \mu \mathrm{l}$ of PI solution at room temperature in the dark for $15 \mathrm{~min}$. Analyses were then performed using a FACSCalibur ${ }^{\mathrm{TM}}$ flow cytometer.

Morphological characteristics of the nucleus by DAPI stain. The cells were first cultured on slides in 24-well plates at a density of $1 \times 10^{4}$ cells/well. After treatment with different agents, the cells on the slides were fixed by incubation in 4\% paraformaldehyde (PFA) for $30 \mathrm{~min}$. After washing with PBS three times, the cells were incubated in $1 \mathrm{mg} / \mathrm{ml}$ DAPI in methanol for $30 \mathrm{~min}$ in the dark. The cells were then observed with a fluorescence microscope.

Western blot analysis. After different treatments, cells were lysed, and the isolated protein was quantified using the Bradford method, subjected to sodium dodecyl sulfate polyacrylamide gel electrophoresis, and then transferred on to a polyvinylidene fluoride membrane. After being blocked, the membrane was incubated with primary polyclonal antibodies of anti-Bax, Bcl-2, caspase-3, caspase-8, caspase-9, survivin and GAPDH overnight at $4^{\circ} \mathrm{C}$, and subsequently incubated with horseradish peroxidase-conjugated $\operatorname{IgG}$ antibody as the secondary antibody for $1 \mathrm{~h}$ at room temperature. The protein bands were detected using an enhanced electrochemiluminescence detection system (ECL system; Amersham Pharmacia Biotech, Amersham, UK). After normalization using the corresponding GAPDH expression, the expression levels of Bax, Bcl-2, caspase-3, caspase-8, caspase-9 and survivin were determined using densitometry scans.

Gastric cancer xenograft model in nude mice. In vivo antitumor efficacy of paclitaxel, nab-paclitaxel and trastuzumab/ nab-paclitaxel were evaluated through tumor-bearing mice. $\mathrm{BALB} / \mathrm{c}$ nude mice were kept in filter-topped cages with standard rodent chow, water available ad libitum, and a 12-h light/dark cycle. The experiment protocol was approved by the Committee on Ethical Animal Experiments at Southeast University. The mice were fed with sterile food in a specific pathogen-free facility. All mice were injected subcutaneously with $5 \times 10^{6}$ NCI-N87 cells. The length (a) and width (b) of the tumor were measured every other day. Tumor volumes $\left(\mathrm{V} / \mathrm{mm}^{3}\right)$ were calculated using the formula: $\mathrm{V}=1 / 2 \times \mathrm{a} \mathrm{x} \mathrm{b}^{2}$. When tumor volumes reached $\sim 60 \mathrm{~mm}^{3}$ (22), the mice were randomly divided into four groups: PBS as control group, paclitaxel, nab-paclitaxel and trastuzumab/nab-paclitaxel (all the groups were treated with equivalent paclitaxel concentration at $20 \mathrm{mg} / \mathrm{kg}$ ). The intravenous treatment was done twice a week for four times. The RTV (relative tumor volume) $=\mathrm{V}_{\mathrm{X}} / \mathrm{V}_{1}$, where $\mathrm{V}_{\mathrm{X}}$ and $\mathrm{V}_{1}$ represent the volumes on day $\mathrm{X}$ and the first day of tumor treatment. The antitumor efficacy of tumor inhibition rate is defined as both of the tumor volume and weight inhibitory rate, which is calculated using the formula: volume inhibitory rate $(\%)=\left(1-\mathrm{RTV}_{\mathrm{a}}\right.$ group $/ \mathrm{RTV}_{\text {average control group) }} \times 100 \%$.

In vivo imaging of NIR-797-labeled trastuzumab/nab-paclitaxel. Firstly, NIR-797-labeld trastuzumab/nab-paclitaxel were synthesised by physical adsorption. Briefly, $1 \mathrm{mg}$ of NIR-797 was added to $1 \mathrm{ml}$ of paclitaxel, nab-paclitaxel and trastuzumab/nab-paclitaxel (5 $\mathrm{mg} / \mathrm{ml}$ paclitaxel equivalent) respectively. After spining at $10 \mathrm{rpm}$ overnight, the suspensions of trastuzumab/nab-paclitaxel were ultracentrifuged at $10,000 \mathrm{rpm}, 4^{\circ} \mathrm{C}$ for $15 \mathrm{~min}$ to remove unadsorpted dye, finally the precipitations were resuspended in $1 \mathrm{ml}$ of PBS for injection. For in vivo imaging, the tumor-bearing mice were injected via tail vein at a single dose of NIR-797-labled paclitaxel, NIR-797-labled nab-paclitaxel and NIR-797-labled trastuzumab/nab-paclitaxel at $20 \mathrm{mg} / \mathrm{kg}$ paclitaxel equivalent concentration when the tumors reached $\sim 60 \mathrm{~mm}^{3}$. The mice were imaged in a small animal imaging system by X-ray and fluorescence at $0,2,4,8,24,48$ and $72 \mathrm{~h}$ after injection.

Histopathological examination. After different treatments, the mice were sacrificed by cervical dislocation. The organs, including lung, heart, liver, spleen, kidney and tumor tissues isolated from each group were immersed in 4\% PFA solution, embedded in paraffin, and stained with hematoxylin-eosin. Thereafter, the tissues were examined using an Olympus IX51 microscope (x200; Olympus Corp., Tokyo, Japan).

Statistical analysis. GraphPad Prism 5.0 (GraphPad software; San Diego, CA, USA) was used for all statistical analysis. The mean $\pm \mathrm{SD}$ was determined for each group in the individual experiments. The Student's t-test was used to determine the significance of differences between different groups. P-values $<0.05$ were considered to indicate statistically significant difference.

\section{Results}

Characterization of trastuzumab/nab-paclitaxel. The scheme for the preparation of trastuzumab/nab-paclitaxel is presented in Fig. 1A. The TEM images in Fig. 1B show the trastuzumab/nab-paclitaxel is spherical in shape and in a suitable size $(139.18 \pm 32.06 \mathrm{~nm})$ measured by DLS (Fig. 1C) for drug delivery. The concentration of paclitaxel of trastuzumab/ nab-paclitaxel by UV spectrophotometry indicates that the concentration of paclitaxel was one-tenth of nab-paclitaxel.

In vitro antitumor efficacy. To determine the antitumor efficacy in vitro, we measured the cytotoxicity following treatment of HER2-postive GC NCI-N87 cells with paclitaxel, nab-paclitaxel and trastuzumab/nab-paclitaxel for $48 \mathrm{~h}$. As shown in Fig. 2A, typical characteristic apoptotic changes, such as chromatin condensation, convoluted nuclei with cavitations, fragmentation of the nucleus, and apoptotic bodies, could be found after different treatments. That is to say, paclitaxel, nab-paclitaxel, and trastuzumab/nab-paclitaxel could inhibit the growth of NCI-N87 cells, whereas trastuzumab/nab-paclitaxel was found to be more cytotoxic than 
$\mathbf{A}$

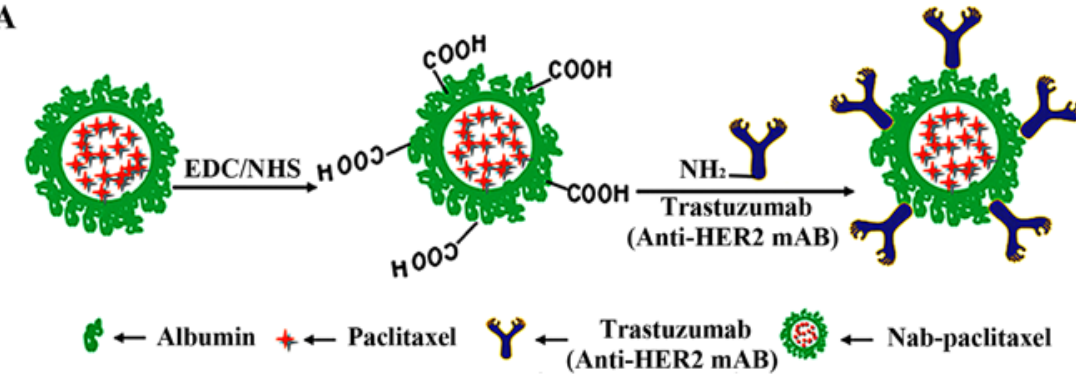

B

C
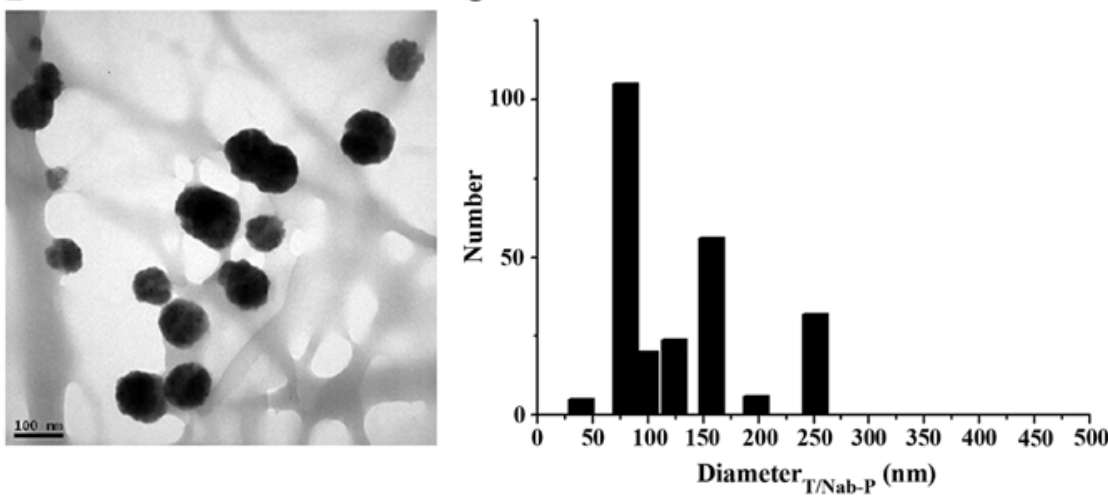

Figure 1. Characterization of trastuzumab/nab-paclitaxel. (A) The scheme for the preparation of trastuzumab/nab-paclitaxel. (B) TEM image. (C) Size distribution.

A
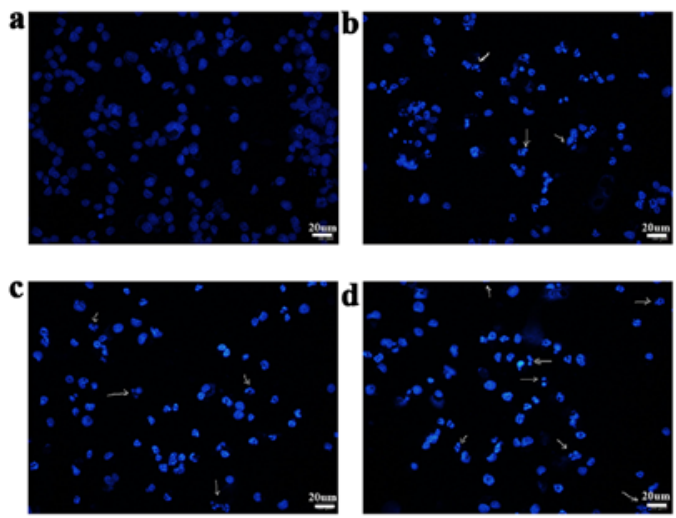
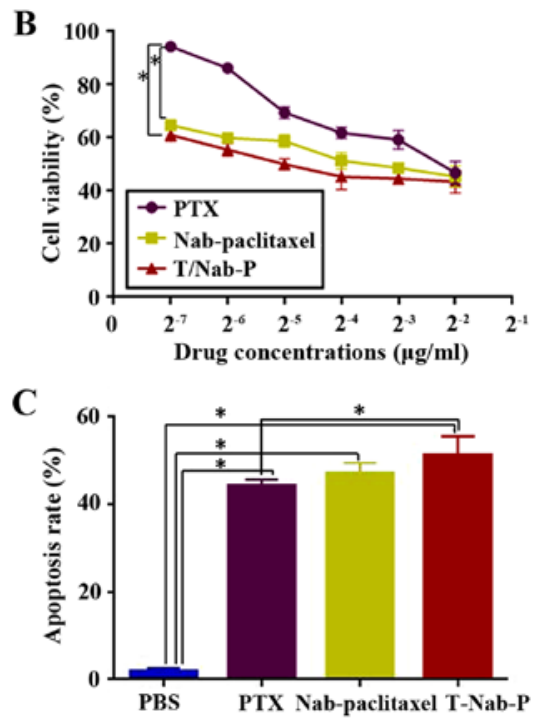

Figure 2. Antitumor efficacy of trastuzumab/nab-paclitaxel in vitro. (A) Morphology of nuclear of NCI-N87 cells treated by paclitaxel, nab-paclitaxel and trastuzumab/nab-paclitaxel. PBS as control (bar, $20 \mu \mathrm{m}$ ). The white arrows indicate chromatin fragmentation of nucleus. (B) Cytotoxic effect of paclitaxel, nab-paclitaxel and trastuzumab/nab-paclitaxel. (C) Apoptosis rate of NCI-N87 cells with DAPI staining by paclitaxel, nab-paclitaxel and trastuzumab/ nab-paclitaxel. PBS as control.

paclitaxel and nab-paclitaxel (Fig. 2B). The half-maximal inhibitory concentration $\left(\mathrm{IC}_{50}\right)$, defined as the concentration of paclitaxel to kill $50 \%$ of cells, was found to be $0.24 \pm 0.08$, $0.13 \pm 0.03$ and $0.048 \pm 0.01 \mu \mathrm{g} / \mathrm{ml}$ for paclitaxel, nab-paclitaxel and trastuzumab/nab-paclitaxel, respectively, with an excellent dose-effect relationship, suggesting that the killing effects of these drugs were dose-dependent. Similarly, the apoptosis rate was higher in trastuzumab/nab-paclitaxel group $(51.30 \pm 2.28 \%)$ than paclitaxel group $(43.32 \pm 1.08 \%)$ and nab-paclitaxel group $(46.64 \pm 1.47 \%)$ in NCI-N87 cells (Fig. 2C). These data clearly suggest that trastuzumab/nab-paclitaxel could enhance cytotoxic effect against NCI-N87 cells better than paclitaxel and nab-paclitaxel in vitro.

Cell cycle distributions of NCI-N87 cells. We quantified the population of NCI-N87 cells in different stages of the cell cycle upon treatments with paclitaxel, nab-paclitaxel and trastuzumab/nab-paclitaxel, respectively, at a concentration of $0.24 \mu \mathrm{g} / \mathrm{ml}$ paclitaxel equivalent for $48 \mathrm{~h}$. In our study, after different treatments, G2/M arrest was frequently observed in 
A
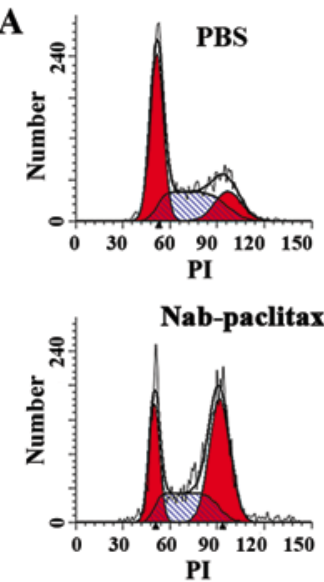
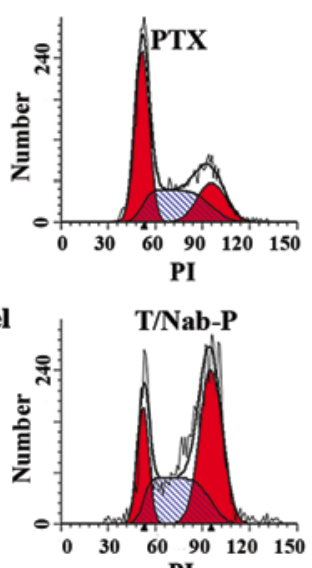

B

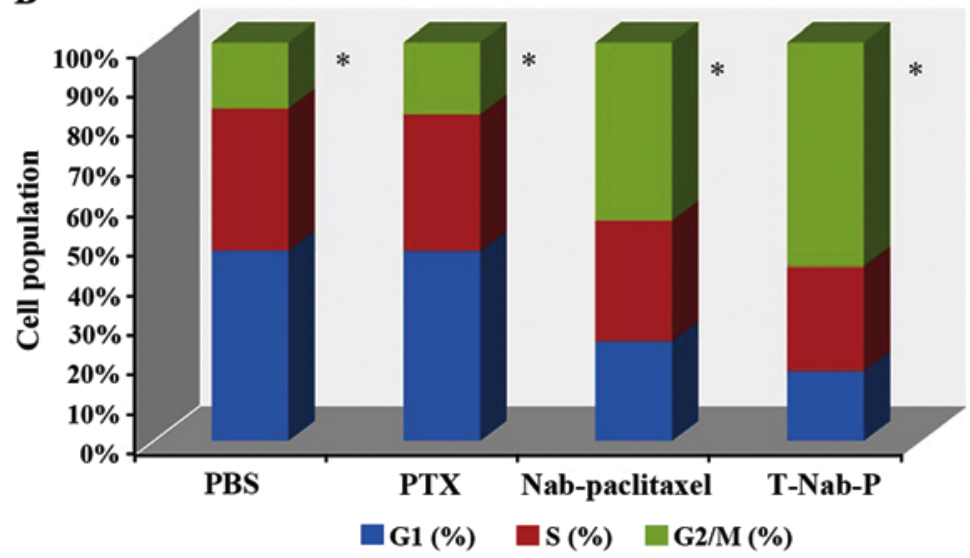

Figure 3. Cell cycle distributions of NCI-N87 cells by different treatments. (A) Cells were exposed to different treatment (PBS, paclitaxel, nab-paclitaxel and trastuzumab/nab-paclitaxel) for $48 \mathrm{~h}$, followed by analysis of cell cycle by flow cytometry. (B) Distribution of NCI-N87 cells at different phases of the cell cycle distribution with different treatment (PBS, paclitaxel, nab-paclitaxel and trastuzumab/nab-paclitaxel).

A
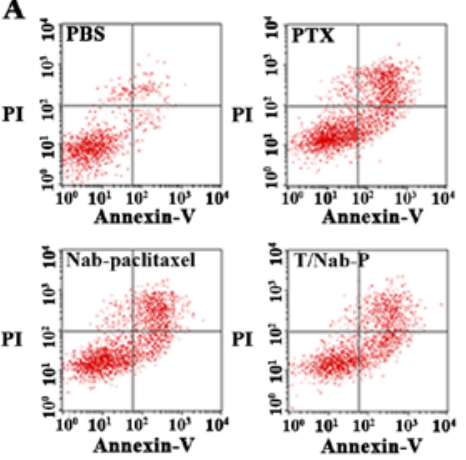

C

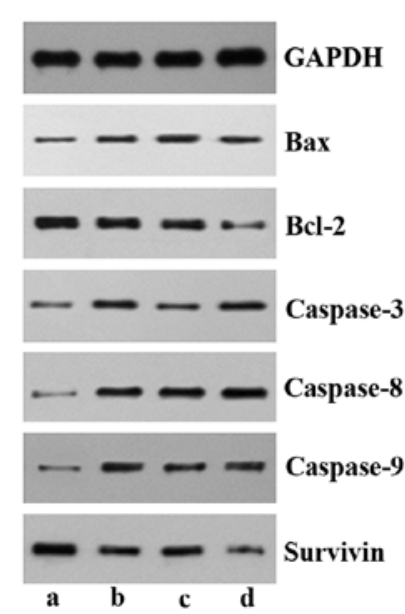

B

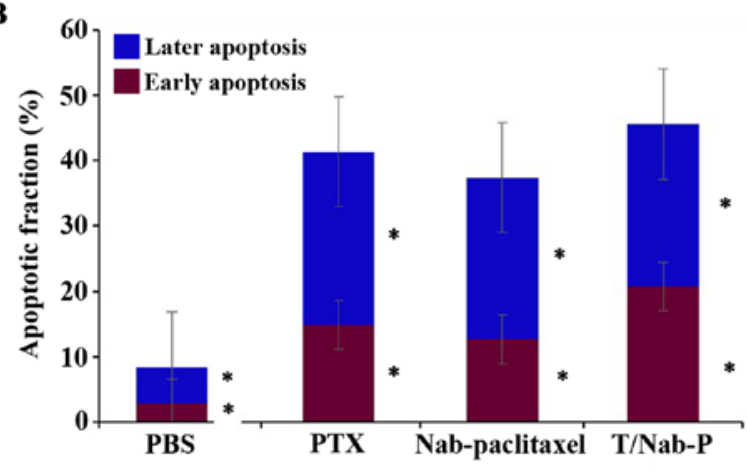

D

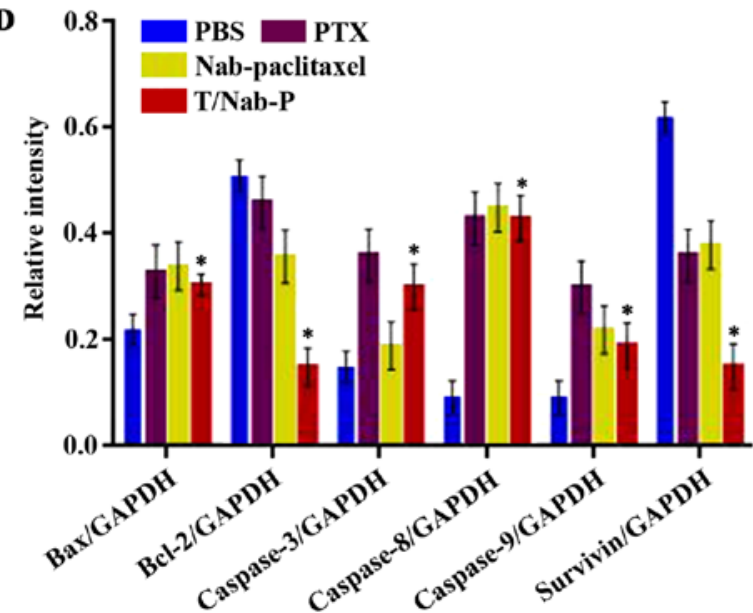

Figure 4. Induction of apoptosis in NCI-N87 cells by different treatments. (A) NCI-N87 cells were treated with paclitaxel, nab-paclitaxel and trastuzumab/ nab-paclitaxel at concentration of $0.24 \mu \mathrm{g} / \mathrm{ml}$ for $48 \mathrm{~h}$, while PBS group was the control. (B) The apoptosis rates are calculated by adding up the percentage of early apoptosis rate and later apoptosis rate. (C) Western blot analysis of endogenous and different treatments (a) PBS, (b) paclitaxel, (c) nab-paclitaxel, (d) trastuzumab/nab-paclitaxel)-induced Bax, Bcl-2, caspase-3, caspase-8, caspase-9 and survivin protein levels in NCI-N87 cells. (D) The relative intensity of Bax, Bcl-2, caspase-3, caspase-8, caspase-9 and survivin protein after 48 -h treatment.

NCI-N87 cells. Moreover, trastuzumab/nab-paclitaxel showed more significant $\mathrm{G} 2 / \mathrm{M}$ arrest (56.35 $\pm 2.14 \%)$ than that of paclitaxel $(18.17 \pm 1.34 \%)$ and nab-paclitaxel $(44.83 \pm 2.58 \%)$, which is shown in Fig. 3.

Apoptosis induction in NCI-N87 cells. Similar to the two clinical drugs paclitaxel and nab-paclitaxel, the novel ANC of trastuzumab/nab-paclitaxel could also induce apoptosis in NCI-N87 cells (Fig. 4A). The early apoptotic rates of NCI-N87 cells treated by trastuzumab/nab-paclitaxel $(20.8 \pm 0.28 \%)$ is higher than that of nab-paclitaxel $(14.9 \pm 0.17 \%)$, paclitaxel $(12.7 \pm 0.65 \%)$, and PBS as control $(2.8 \pm 0.12 \%)$ (Fig. 4B). To explore the possible signaling pathways involved in apoptosis, we examined the changes of the apoptosis-related proteins, 
A

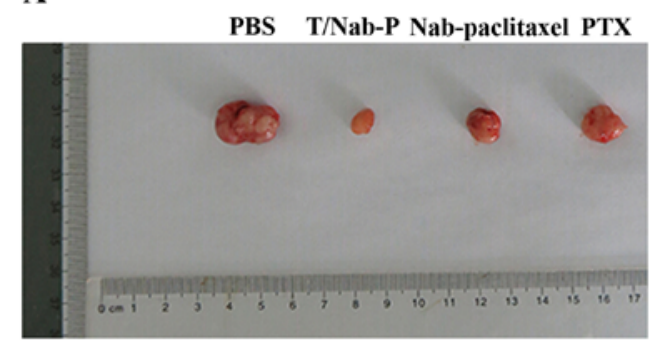

$\mathrm{C}$

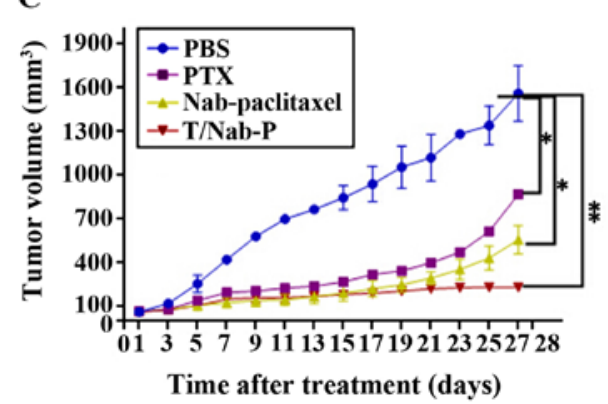

B
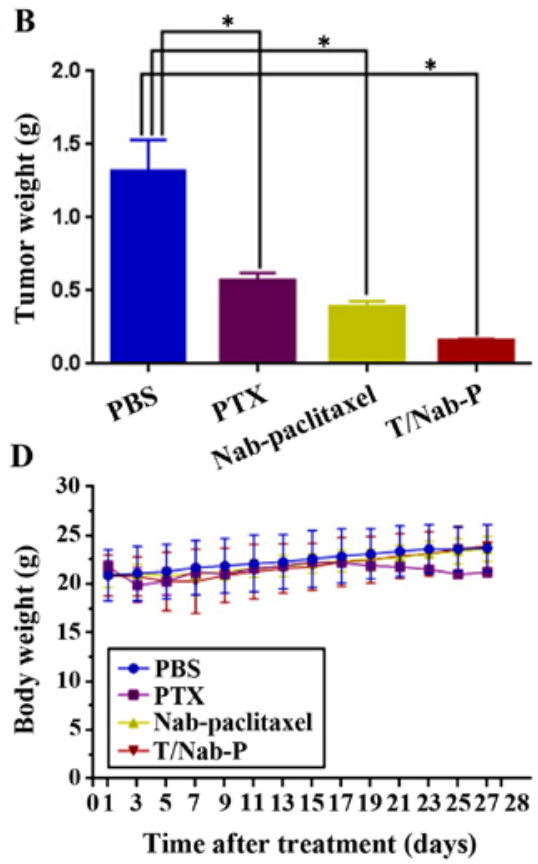

Figure 5. The antitumor effect of different treatments on NCI-N87 human xenograft models. (A) At the experimental end-point, tumors were dissected and photographed ( $\mathrm{n}=3$ per group). Only one mouse is demonstrated as an example for each group. (B) The tumor weight were compared with Student's t-test $\left(\mathrm{n}=3,{ }^{*} \mathrm{P}<0.05\right)$. (C) The mice transplanted with NCI-N87 xenograft were randomly divided into two groups and given injection of trastuzumab/nab-paclitaxel compared with paclitaxel, nab-paclitaxel and PBS $(20 \mathrm{mg} / \mathrm{kg}$ per week for a period of 4 weeks). The tumor volumes were expressed as mean \pm SD ( $=3$, $\left.{ }^{*} \mathrm{P}<0.05,{ }^{* *} \mathrm{P}<0.01\right)$. (D) The average body weight of each group was expressed as mean $\pm \operatorname{SD}(\mathrm{n}=3)$.

including caspase-3, caspase-8, caspase-9, Bax, Bcl-2, and survivin by western blotting. As shown in Fig. 4C and D, when treated with paclitaxel, nab-paclitaxel and trastuzumab/ nab-paclitaxel for $48 \mathrm{~h}$, the levels of both Bcl-2 and survivin in NCI-N87 cells were significantly downregulated, while those of Bax, caspase-3, caspase- 8 , caspase- 9 protein were upregulated as well as the ratio of $\mathrm{Bax} / \mathrm{Bcl}-2$.

In vivo antitumor efficacy. Antitumor efficacy of trastuzumab/ nab-paclitaxel compared with paclitaxel and nab-paclitaxel were further evaluated in NCI-N87 xenograft models at a $20 \mathrm{mg} / \mathrm{kg}$ paclitaxel equivalent dose in vivo. During the experiment period, all the mice were weighed and tumor volumes were measured every other day. At 4 weeks after treatment, mice were sacrificed. Then the mouse tumors were imaged (Fig. 5A) and tumor weight was recorded (Fig. 5B). Mean tumor volume treated with trastuzumab/nab-paclitaxel was $233 \pm 24 \mathrm{~mm}^{3}$, nab-paclitaxel of $559 \pm 97 \mathrm{~mm}^{3}$, paclitaxel of $871 \pm 94 \mathrm{~mm}^{3}$ and PBS as control of $1,576 \pm 190 \mathrm{~mm}^{3}$ (Fig. 5C). Obvious significant tumor regression was obtained in mice treated with trastuzumab/nab-paclitaxel compared with paclitaxel and nab-paclitaxel. In addition, trastuzumab/ nab-paclitaxel compared with nab-paclitaxel and paclitaxel did not have significanly increased body weight (Fig. 5D).

NIRF imaging and biodistribution of NIR-797-labeled drugs in tumor-bearing mice. To visualize the biodistribution of trastuzumab/nab-paclitaxel in NCI-N87 tumor-bearing mice, a near-infrared fluorescent (NIRF) dye, NIR-797, was labeled to paclitaxel, nab-paclitaxel and trastuzumab/nab-paclitaxel. NIR fluorescence signals were clearly and dynamically observed in the mice. As shown in Fig. 6,2 hafter injection, these fluorescence signals of NIR-797-labeled paclitaxel were strong and mainly localized in the body area, but it began to decrease after $8 \mathrm{~h}$ and decreased gradually especially in the tumor site with no distribution in the brain. Obvious increased accumulation of NIR fluorescence signals was observed in the tumor site of the mice injected nab-paclitaxel and trastuzumab/nab-paclitaxel. What is inspiring, as time increased, the fluorescence signals of tumors became stronger and the fluorescence signals of liver began to weaken. Fluorescence signals of NIR-797-labeled trastuzumab/nab-paclitaxel remains strong until $48 \mathrm{~h}$ after injection, at which point the fluorescence signals of NIR-797labeled nab-paclitaxel has already decreased. In addition, $72 \mathrm{~h}$ after injection, fluorescence signals could only be seen in the liver and tumor site which indicated a better targeting and sustained release of trastuzumab/nab-paclitaxel. Furthermore, these fluorescence signals of trastuzumab/nab-paclitaxel group were only observed in liver and tumor areas.

Histopathological examination. We carried out histological bioanalysis of organs to evaluate the potential side effects of trastuzumab/nab-paclitaxel on the main organs of mice in vivo. As shown in Fig. 7, there were no apparent histopathologic changes in the tissues, including heart, liver, spleen, lung and kidney.

\section{Discussion}

AGC remains one of the most lethal malignancies due to its intrinsic resistance and its aggressiveness to standard chemotherapy and targeted therapy. Nab-paclitaxel as carriers of chemotherapeutic drugs to reverse the toxicity of paclitaxel have displayed that nab-paclitaxel can significantly increase 


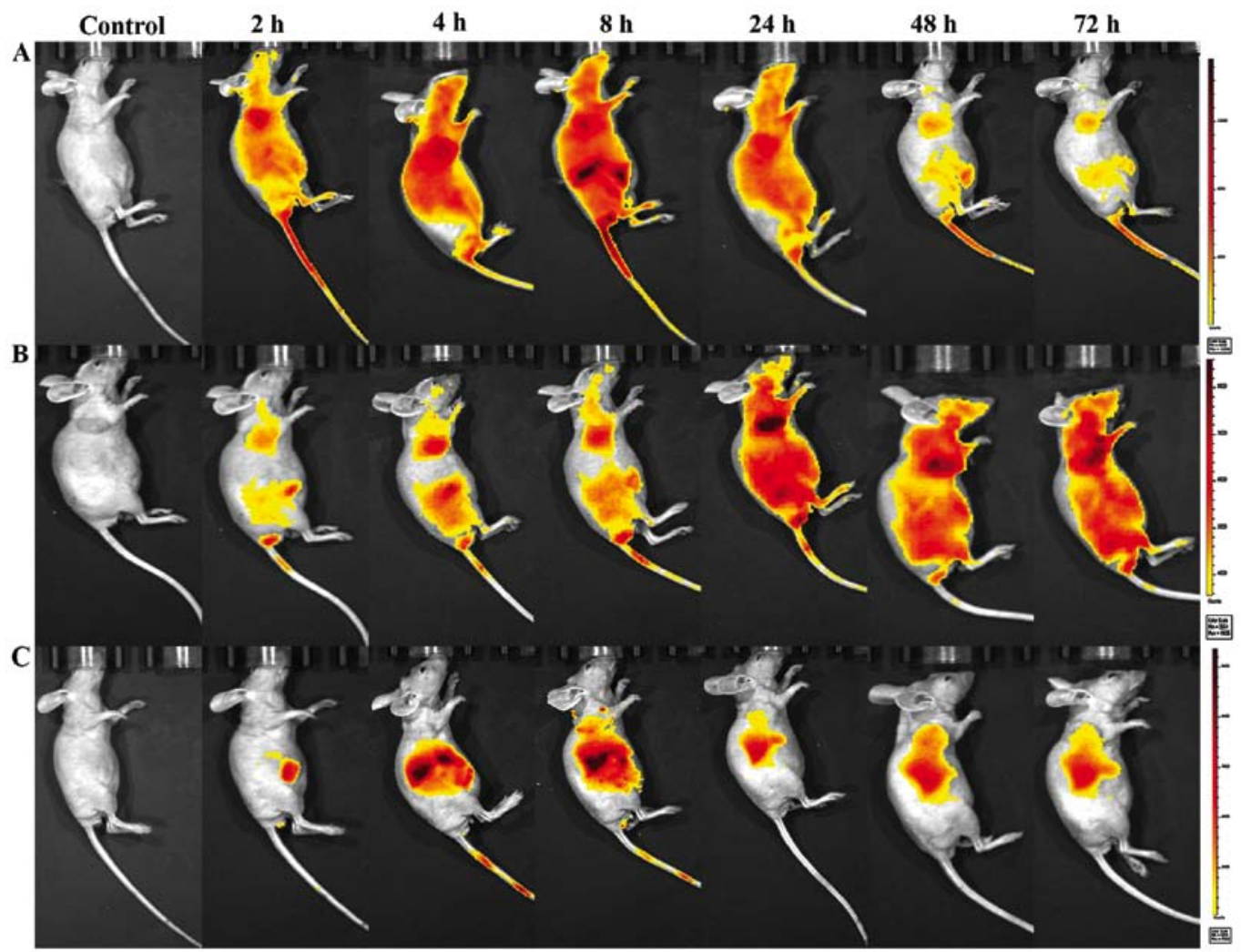

Figure 6. NIRF images of NCI-N87 tumor-bearing mice after treatments. (A) NIR797-labeled paclitaxel. (B) NIR797-labeled nab-paclitaxel. (C) NIR797labeled trastuzumab/nab-paclitaxel. The color depth in tumor tissue indicates the drug concentration in that area.

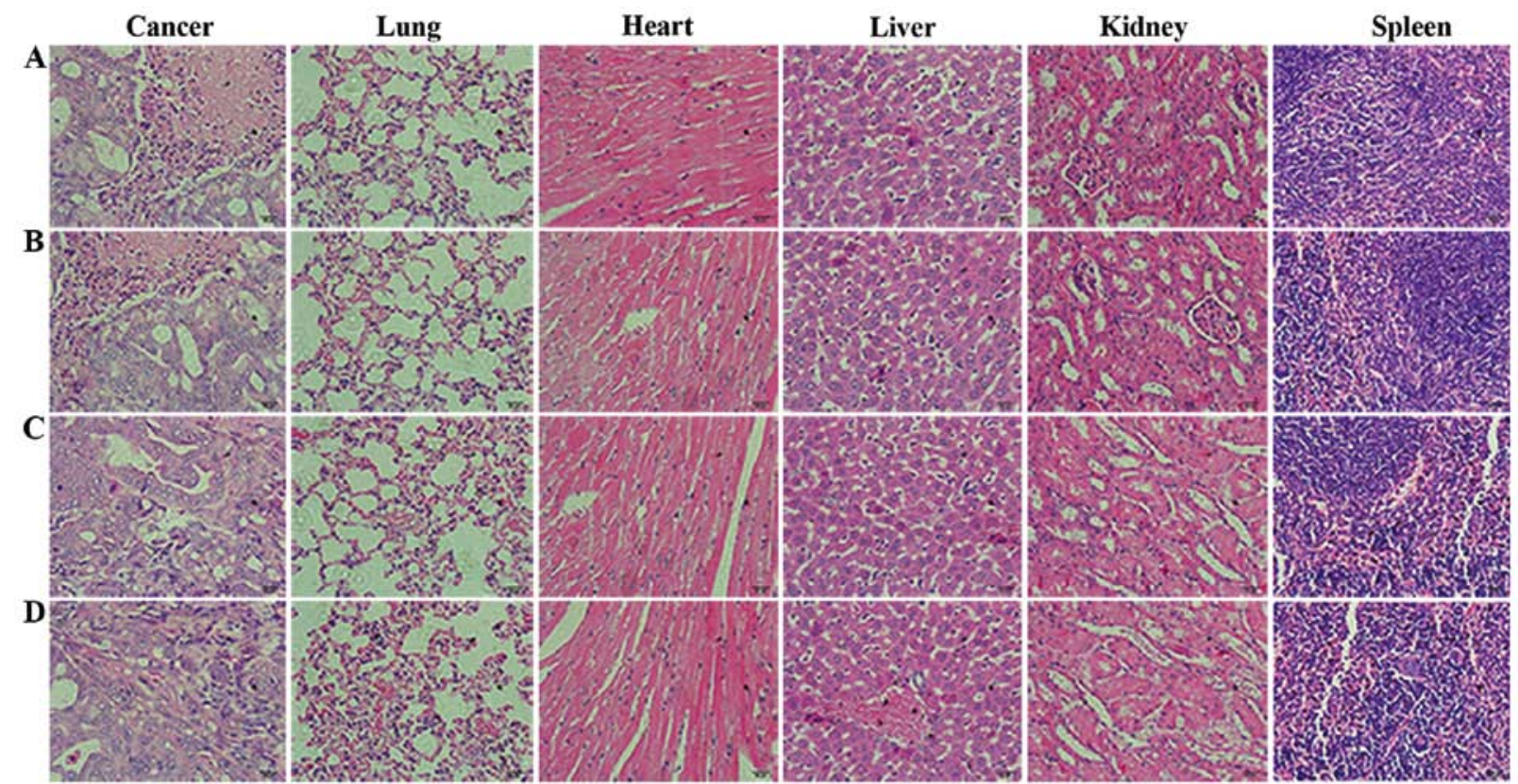

Figure 7. Histopathological examination of organs and tumor tissues in NCI-N87 xenograft model after the treatment (hematoxylin-eosin staining, x200). (A) PBS. (B) Paclitaxel. (C) Nab-paclitaxel. (D) Trastuzumab/nab-paclitaxel.

the antitumor effection and still maintain a certain concentration. In clinic, trastuzumab combined with chemotherapy as ADC demonstrates a significant survival benefit in patients with HER2-positive AGC. Thus, in this study, we further constructed the ANC of trastuzumab/nab-paclitaxel for AGC.
Apoptosis is the preferred anticancer manner regulated by genes (23-25). Many oncogenes and tumor suppressor genes are involved in the regulation of apoptosis; the proto-oncogene $\mathrm{Bcl}-2$ is the most important (26). Bcl-2 and Bax are a pair of positive and negative regulators of genes; Bax induced apoptosis, while Bcl-2 inhibited apoptosis (27). Caspase-3 may 
promote apoptosis, while survivin is the strongest inhibitor of apoptosis in AGC, also promoting cell proliferation, which can directly inhibit the activity of caspase- 3 and caspase- 8 and caspase-9, thereby blocking the apoptotic process $(28,29)$. These results of apoptosis phenomenon in NCI-N87 cells treated with PBS, paclitaxel, nab-paclitaxel and trastuzumab/ nab-paclitaxel by western blotting observation showed that ANC of trastuzumab/nab-paclitaxel could decrease Bcl-2 and survivin protein expression, and increase Bax and caspase-3 protein expression; the difference was more significant than the control group. Caspase activation is generally considered to be the hallmark of apoptosis, and caspase- 3 is the main effector caspase that is involved in apoptosis. These results indicate that trastuzumab/nab-paclitaxel could induce anticancer activity in a caspase-dependent manner, and effectiveness of the survivin pathway.

Paclitaxel's mode of antitumor action is the disruption of microtubule dynamics, and paclitaxel is believed to mediate $\mathrm{G} 2 / \mathrm{M}$ cell cycle arrest in various cancer cells, including GC (30-32). In our study, after different treatments, G2/M arrest was frequently observed in NCI-N87 cells. Furthermore, trastuzumab/nab-paclitaxel showed more significant $\mathrm{G} 2 / \mathrm{M}$ arrest $(56.35 \pm 2.14 \%)$ than that of paclitaxel $(18.17 \pm 1.34 \%)$ and nab-paclitaxel $(44.83 \pm 2.58 \%)$. Conjugation of paclitaxel and trastuzumab as an ADC was thought to be promising and worthy $(33,34)$. However, clinical results from ADC failed to demonstrate therapeutic benefit with shortcomings of poor in vitro potency, modest in vivo activity and localization in human tumors (35). Albumin-bound formulation for paclitaxel based on nano-drug delivery system, nab-paclitaxel, has the advantage of passive target for tumor due to EPR effect. Now, deliberate modifications of ligand or antibody to the surface of nanoparticles were conducted to achieve specific targeting to the corresponding receptor on tumor cells $(22,36)$. The unique targeting mechanism of HSA $(22,37)$ and passive targeting property of nab-paclitaxel along with the active targeting property of anti-HER 2 antibody may introduce sequentially dual-targeting and more precise paclitaxel delivery.

Currently, the NIRF imaging technique is widely used for cancer molecular imaging research; it has a clearer and longer visualization for tracking in vivo. In our study, fluorescence signals of NIR-797-labeled trastuzumab/nab-paclitaxel remains strong until $48 \mathrm{~h}$ after injection. Furthermore, the fluorescence signals of trastuzumab/nab-paclitaxel group were only observed in liver and tumor area, illustrating that the ANC of trastuzumab/nab-paclitaxel could target the tumor tissue more precisely than nanoparticles. In vivo antitumor efficacy demonstrated that the ANC of the trastuzumab/nab-paclitaxel had more significant tumor regression than other treatment groups. In addition, trastuzumab/nab-paclitaxel compared with nab-paclitaxel and paclitaxel had no obviously effect on the quality of life.

In conclusion, this study successfully synthesized antibody-nanoparticle conjugate of trastuzumab/nab-paclitaxel with properties of passive and active target. In vitro and in vivo findings illustrated that trastuzumab/nab-paclitaxel could enhance antitumor efficacy, which could represent a novel precisely targeting therapeutic agent for HER2-positive GC.

\section{Acknowledgements}

This study was supported by the National Nature Science Foundation of People's Republic of China (81371678).

\section{References}

1. Siegel RL, Miller KD and Jemal A: Cancer statistics, 2016. CA Cancer J Clin 66: 7-30, 2016.

2. Chen W, Zheng R, Baade PD, Zhang S, Zeng H, Bray F, Jemal A, Yu XQ and He J: Cancer statistics in China, 2015. CA Cancer J Clin 66: 115-132, 2016.

3. Yu B and Xie J: Identifying therapeutic targets in gastric cancer: The current status and future direction. Acta Biochim Biophys Sin (Shanghai) 48: 90-96, 2016

4. Sakai D, Satoh T, Kurokawa Y, Kudo T, Nishikawa K, Oka Y, Tsujinaka T, Shimokawa T, Doki Y and Furukawa H: A phase II trial of trastuzumab combined with irinotecan in patients with advanced HER2-positive chemo-refractory gastric cancer: Osaka Gastrointestinal Cancer Chemotherapy Study Group OGSG1203 (HERBIS-5). Jpn J Clin Oncol 43: 838-840, 2013.

5. Gumusay O, Benekli M, Ekinci O, Baykara M, Ozet A, Coskun U, Demirci U, Uner A, Dursun A, Atak EY, et al: Discordances in HER2 status between primary gastric cancer and corresponding metastatic sites. Jpn J Clin Oncol 45: 416-421, 2015.

6. Bang YJ, Van Cutsem E, Feyereislova A, Chung HC, Shen L, Sawaki A, Lordick F, Ohtsu A, Omuro Y, Satoh T, et al; ToGA Trial Investigators: Trastuzumab in combination with chemotherapy versus chemotherapy alone for treatment of HER2-positive advanced gastric or gastro-oesophageal junction cancer (ToGA): A phase 3, open-label, randomised controlled trial. Lancet 376: 687-697, 2010.

7. Corrigan PA, Cicci TA, Auten JJ and Lowe DK: Ado-trastuzumab emtansine: A HER2-positive targeted antibody-drug conjugate. Ann Pharmacother 48: 1484-1493, 2014.

8. Ahn ER, Wang E and Glück S: Is the improved efficacy of trastuzumab and lapatinib combination worth the added toxicity? A discussion of current evidence, recommendations, and ethical issues regarding dual HER2-targeted therapy. Breast Cancer (Auckl) 6: 191-207, 2012.

9. Moghaddas A and Borhani A: Whether HER2-positive nonbreast cancers are candidates for treatment with Ado-trastuzumab emtansine? J Res Pharm Pract 5: 227-233, 2016.

10. Barok M, Tanner M, Köninki K and Isola J: Trastuzumab-DM1 is highly effective in preclinical models of HER2-positive gastric cancer. Cancer Lett 306: 171-179, 2011.

11. Yamashita-Kashima Y, Shu S, Harada N and Fujimoto-Ouchi K: Enhanced antitumor activity of trastuzumab emtansine (T-DM1) in combination with pertuzumab in a HER2-positive gastric cancer model. Oncol Rep 30: 1087-1093, 2013.

12. Shitara K and Ohtsu A: Advances in systemic therapy for metastatic or advanced gastric cancer. J Natl Compr Canc Netw 14: 1313-1320, 2016.

13. Gupta N, Hatoum H and Dy GK: First line treatment of advanced non-small-cell lung cancer - specific focus on albumin bound paclitaxel. Int J Nanomed 9: 209-221, 2014.

14. Yamamoto T, Miyazaki T, Kurashima Y, Ohata K, Okawa M, Tanaka S, Uenishi T and Ohno K: A case report of successful chemotherapy with tegafur/gimeracil/oteracil and nab-paclitaxel for gastric cancer with chronic renal failure. Gan To Kagaku Ryoho 42: 735-738, 2015 (In Japanese).

15. Vogel A, Kullmann F, Kunzmann V, Al-Batran SE, Oettle H, Plentz R, Siveke J, Springfeld C and Riess H: Patients with advanced pancreatic cancer and hyperbilirubinaemia: Review and German expert opinion on treatment with nab-paclitaxel plus Gemcitabine. Oncol Res Treat 38: 596-603, 2015.

16. Hirsh V: nab-paclitaxel for the management of patients with advanced non-small-cell lung cancer. Expert Rev Anticancer Ther 14: 129-141, 2014.

17. Von Hoff DD, Ervin T, Arena FP, Chiorean EG, Infante J, Moore M, Seay T, Tjulandin SA, Ma WW, Saleh MN, et al: Increased survival in pancreatic cancer with nab-paclitaxel plus gemcitabine. N Engl J Med 369: 1691-1703, 2013.

18. Montero AJ, Adams B, Diaz-Montero CM and Glück S: nab-paclitaxel in the treatment of metastatic breast cancer: A comprehensive review. Expert Rev Clin Pharmacol 4: 329-334, 2011. 
19. Ruttala HB, Ramasamy T, Shin BS, Choi HG, Yong CS and Kim JO: Layer-by-layer assembly of hierarchical nanoarchitectures to enhance the systemic performance of nanoparticle albumin-bound paclitaxel. Int J Pharm 519: 11-21, 2017.

20. van Lith SA, van Duijnhoven SM, Navis AC, Leenders WP, Dolk E, Wennink JW, van Nostrum CF and van Hest JC: Legomedicine - A versatile chemo-enzymatic approach for the preparation of targeted dual-labeled llama antibody-nanoparticle conjugates. Bioconjug Chem 28: 539-548, 2017.

21. Obaid G, Chambrier I, Cook MJ and Russell DA: Cancer targeting with biomolecules: A comparative study of photodynamic therapy efficacy using antibody or lectin conjugated phthalocyanine-PEG gold nanoparticles. Photochem Photobiol Sci 14: 737-747, 2015.

22. Chen L, Chen F, Zhao M, Zhu X, Ke C, Yu J, Yan Z, Zhang F, Sun Y, Chen D, et al: A redox-sensitive micelle-like nanoparticle self-assembled from amphiphilic adriamycin-human serum albumin conjugates for tumor targeted therapy. Biomed Res Int 2015: 987404, 2015

23. Su LY, Shi YX, Yan MR, Xi Y and Su XL: Anticancer bioactive peptides suppress human colorectal tumor cell growth and induce apoptosis via modulating the PARP-p53-Mcl-1 signaling pathway. Acta Pharmacol Sin 36: 1514-1519, 2015.

24. Moon JY, Cho M, Ahn KS and Cho SK: Nobiletin induces apoptosis and potentiates the effects of the anticancer drug 5 -fluorouracil in p53-mutated SNU-16 human gastric cancer cells. Nutr Cancer 65: 286-295, 2013.

25. Korbakis D and Scorilas A: Quantitative expression analysis of the apoptosis-related genes BCL2, BAX and BCL2L12 in gastric adenocarcinoma cells following treatment with the anticancer drugs cisplatin, etoposide and taxol. Tumour Biol 33: 865-875, 2012.

26. Lindsay J, Esposti MD and Gilmore AP: Bcl-2 proteins and mitochondria - specificity in membrane targeting for death. Biochim Biophys Acta 1813: 532-539, 2011.

27. Gonzalez-Campora R, Davalos-Casanova G, Beato-Moreno A, Garcia-Escudero A, Pareja Megia MJ, Montironi R and Lopez-Beltran A: BCL-2, TP53 and BAX protein expression in superficial urothelial bladder carcinoma. Cancer Lett 250: 292-299, 2007

28. Sriramoju B, Kanwar RK and Kanwar JR: Nanoformulated cellpenetrating survivin mutant and its dual actions. Int J Nanomed 9: 3279-3298, 2014.
29. Altieri DC: Survivin, cancer networks and pathway-directed drug discovery. Nat Rev Cancer 8: 61-70, 2008.

30. Toiyama Y, Tanaka K, Konishi N, Mohri Y, Tonouchi H, Miki C and Kusunoki M: Administration sequence-dependent antitumor effects of paclitaxel and 5-fluorouracil in the human gastric cancer cell line MKN45. Cancer Chemother Pharmacol 57: 368-375, 2006.

31. Matsuhashi N, Saio M, Matsuo A, Sugiyama Y and Saji S: Apoptosis induced by 5-fluorouracil, cisplatin and paclitaxel are associated with p53 gene status in gastric cancer cell lines. Int J Oncol 26: 1563-1567, 2005.

32. Arranja A, Gouveia LF, Gener P, Rafael DF, Pereira C, Schwartz S Jr and Videira MA: Self-assembly PEGylation assists SLN-paclitaxel delivery inducing cancer cell apoptosis upon internalization. Int J Pharm 501: 180-189, 2016.

33. Bender B, Leipold DD, Xu K, Shen BQ, Tibbitts $\mathrm{J}$ and Friberg LE: A mechanistic pharmacokinetic model elucidating the disposition of trastuzumab emtansine (T-DM1), an antibodydrug conjugate $(\mathrm{ADC})$ for treatment of metastatic breast cancer. AAPS J 16: 994-1008, 2014.

34. Krop IE, Lin NU, Blackwell K, Guardino E, Huober J, Lu M, Miles D, Samant M, Welslau M and Diéras V: Trastuzumab emtansine (T-DM1) versus lapatinib plus capecitabine in patients with HER2-positive metastatic breast cancer and central nervous system metastases: A retrospective, exploratory analysis in EMILIA. Ann Oncol 26: 113-119, 2015.

35. Elzoghby AO, Samy WM and Elgindy NA: Protein-based nanocarriers as promising drug and gene delivery systems. J Control Release 161: 38-49, 2012.

36. Ruan J, Ji J, Song H, Qian Q, Wang K, Wang C and Cui D Fluorescent magnetic nanoparticle-labeled mesenchymal stem cells for targeted imaging and hyperthermia therapy of in vivo gastric cancer. Nanoscale Res Lett 7: 309, 2012.

37. Regino CAS, Ogawa M, Alford R, Wong KJ, Kosaka N, Williams M, Feild BJ, Takahashi M, Choyke PL and Kobayashi H: Two-step synthesis of galactosylated human serum albumin as a targeted optical imaging agent for peritoneal carcinomatosis. J Med Chem 53: 1579-1586, 2010. 